\title{
Experimental Investigation of the Performance of a Feedback Tension Control System Designed for Warping Machines
}

\author{
Recep Eren, Merve İhtiyar, Özge Çelik \\ Bursa Uludag University, Faculty of Engineering, Textile Engineering Department, Gorukle Campus 16059 Nilüfer, Bursa, Turkey
}

Corresponding Author: Recep Eren, erecep@uludag.edu.tr

\begin{abstract}
This paper presents a research work which investigates experimentally the performance of a tension control system realized by a controlled disc brake. An experimental set-up was established by using a creel with step motor-controlled disc brake, laser distance sensor, yarn tension sensor and a 2-unit winding machine. A software was developed in $\mathrm{C}$ programming language to read yarn tension and bobbin diameter data and then to control the disc brake by step motor drive. Experimental investigation was carried out with three different cotton yarn counts and unwinding speeds. It was shown that yarn tension changed from full to empty bobbin at a significant amount depending mainly on yarn number and unwinding speed. The feedback tension control system based on adjusting level of braking responded well to short, medium and long term tension variations and enabled unwinding tension control mostly within $\pm 1 \mathrm{cN}$ deviation from the adjusted value.
\end{abstract}

\section{ARTICLE HISTORY}

Received: 10.04.2019

Accepted: 22.10.2019

\section{KEYWORDS}

warping, tension control, creel, tension measurement, weaving preparation

\section{INTRODUCTION}

Warping is an important preparation process before weaving and directly affects the efficiency of weaving process and quality of woven fabrics. Sectional warping is most widely used warping method among the others and warping tension is a critical parameter for a good quality warp preparation especially with increasing warping speeds. As it is well known, yarn tension shows a significant increase with decreasing bobbin diameter especially at last one third part of full bobbin diameter during unwinding. This tension change becomes more severe and deteriorates the warping quality at increasing warping speeds. Warp is wound on a conical drum in sections side by side in sectional warping machine until the total number of warp yarn is wound. Any tension change between sections causes winding of warp sections at different diameters and therefore different lengths. During transfer of the warp from conical drum to warp beam (i.e., during beaming process), sections of different diameters are wound at different tension and this will produce irregular dyed sections in woven fabrics.

At increasing warping speeds, tension-controlled creels are required to keep the warping tension at the adjusted value irrespective of bobbin diameter for a good quality warp preparation. Tension to yarn can be given by two methods. First method has long been used in textile machines and processes and applies tension to yarns by friction. Friction is applied to yarns either by drawing yarns between two metal plates (additive) or by wrapping around certain number of cylindrical rods (multiplying type) [1]. These mechanical elements are called yarn brakes or yarn tensioners. In many yarn brakes, a combination of additive and multiplying methods is used. Weigh or spring force is employed to adjust tension in additive type yarn tensioners and wrapping angle is adjusted to apply the desired yarn tension in multiplying type of tensioners. Second method

To cite this article: Eren R, İhtiyar M, Çelik Ö. 2019. Experimental investigation of the performance of a feedback tension control system designed for warping machines. Tekstil ve Konfeksiyon 29(4), 289-299. 
for applying tension to a yarn is to adjust the yarn feeding speed. As yarn is drawn at certain speed in warping, its tension is determined by yarn feeding speed from a bobbin in the creel. Most of the tension-controlled creels employ yarn brakes or tensioners using the first method. Individual yarn tension-controlled creel uses the second tensioning method where tension of each individual warp yarn is measured and yarn feeding speed is adjusted by a feedback tension control system to keep yarn tension at the adjusted value during warping process irrespective of bobbin diameter. There are as many feedback tension control systems as the number of bobbins in the creel. As there are as many tension sensors and yarn feeding motors as number of bobbins in the creel, it is more expensive tension control method. But it minimizes tension differences between warp yarns in a section and provides more precise tension control. Individual tension control system is available in commercially [2].

When the literature is reviewed, there are mainly patents explaining warp tension control in warping creels and its effect on warping quality. There are also some researches related to yarn tension in warping creels and its effect on warping quality and warp properties. Fernando and Kuruppu analyzed theoretically warp yarn tension change in warping creel during unwinding from conical bobbins [3]. They expressed mathematical relationships for different regions of the creel and calculated yarn tension for 20 and 40 tex warp yarns at 200 and $400 \mathrm{~m} / \mathrm{min}$ speeds. Mathematical calculations showed that yarn tension changed significantly at different regions of warping creel from unwinding to warping drum. Yarn tension at yarn guide in unwinding region was calculated by the mathematical expression recommended by Isakov [4]. After evaluating the results, they recommended to use a tension controlled warping creel for a good quality and homogeneous warp preparation. Dorgham investigated experimentally the effect of warping speed and warp tension on warp yarn properties like breaking strength and breaking elongation [5]. Warps were prepared from polyester filaments with three yarn numbers $(70,150,300$ denier) and each with three applied tensions $(0.15,0.22$, $0.29 \mathrm{cN} /$ dtex) at $200,400,600 \mathrm{~m} / \mathrm{min}$ warping speeds. Tensile strength and elongation at break were measured. The results of measurements showed optimum tensile strength at warping speed of $400 \mathrm{~m} / \mathrm{min}$ under a tension of $0.22 \mathrm{cN} /$ dtex. In addition, the optimum elongation percentage was obtained at warping speed of $200 \mathrm{~m} / \mathrm{min}$, under a tension of $0.22 \mathrm{cN} /$ dtex for 70 denier yarns. For 150 and 300 denier yarns, the optimum tensile strength and elongation percentage were measured at warping speed of $600 \mathrm{~m} / \mathrm{min}$ under a tension of $0.29 \mathrm{cN} / \mathrm{dtex}$ [5]. Beerli and Guntli explained an apparatus and a method to control yarn tension in the creel of a sectional warping machine. The creel had a central drive motor for adjusting amount of braking in yarn brakes. Section warp tension was measured in the measuring roller unit just before the winding on cone drum and compared with the desired value. Depending on the difference between them, yarn brakes of all units were adjusted by motor drive centrally to keep tension of warp sections constant [6]. Zeller et al explained a process for the operation of a bobbin creel. In addition to warp sheet tension measurement in each warp section, they measured tension of each individual warp end by a separate tension sensor. They also used an individual step motor drive for yarn brake of each bobbin. Yarn tension sensors were positioned at the front of creel in rows of 8 sensors. Before yarn brakes, yarn tensioners of rod and crepe type were used for pre-tensioning. Yarn tensioners were also able to be controlled by electric motors centrally or individually like yarn brakes. Yarn tensioners also prevented entanglements due to high yarn twist. According to invention in the patent, yarn tension of each individual warp was measured and the measured tension was compared with the desired tension. Pressure of yarn brake was adjusted by the step motor of each unit. In this way, irrespective of yarn length and bobbin position in the creel always the same yarn tension was obtained. Warp tension was also measured in sections and when there was a deviation between the set and the measured values, yarn brakes of all bobbins were adjusted. It was recommended in the patent that yarn tension would be measured at a position as close to winding unit as possible, for example between leasing reed and measuring rollers. Conventional controlled additive type of yarn brakes were used in the creel [7]. Kleiner and Jakop explained a method and a device for regulating and controlling warp yarn tension in warping creels. Brake unit or tensioning element of each bobbin was controlled by a separate motor. Apart from disc or rod type of brakes, a yarn wheel driven by a separate motor was used as a yarn tensioning element in which yarn was wrapped certain number of revolutions around the wheel and then taken to the winding unit after passing over a tension measurement sensor. A separate tension sensor was used for each warp yarn in the creel. In this way, as many feedback tension control systems as the number bobbins in the creel were formed and yarn tension was controlled and regulated during normal running in this way. The same control algorithms were not found sufficient for the start and stop periods of a warping machine. A different control method was explained in the patent based on winding speed of warp to drum for the start and stop periods. Based on winding speed change, acceleration of yarn, yarn tension, drive motor inertia and frictional forces affecting the motor were determined and total motor torque required during start and stop periods of a warping machine were calculated. By adjusting motor current during start and stop periods, yarn tension deviations from the set value were minimized. Individual yarn tension control also minimized tension differences between warp yarns of different lengths [8]. Baba explained a tension control method for warping machines in which he combined yarn speed control and breaking force adjustment. He conducted experiments at $800 \mathrm{~m} / \mathrm{min}$ unwinding speed with $\mathrm{Ne} 40 / 1$ cotton yarn and measured bobbin diameter together with yarn tension. He did not mention any other parameter regarding his measurements. He showed that yarn tension decreased up to 
certain diameter starting from full bobbin and then after a minimum it turned to increase. Towards the empty bobbin, yarn tension showed a very sharp increase. He also showed a linear increase in yarn tension with yarn winding speed between 500 and $800 \mathrm{~m} / \mathrm{min}$ corresponding around to $5.5 \mathrm{gr}$ tension change. Baba explained a tension control approach based on adjusting yarn braking force at bobbin diameters relatively larger. As yarn tension showed increase above the adjusted value even after yarn brake is put out of action, he decreased winding speed to keep yarn tension constant during winding [9].

Although there are patents in the literature related to tension control systems in warping creels, no published research has been found in the literature investigating theoretically or experimentally the performance and limitations of tension control systems in warping creels. This paper presents an experimental research explaining the performance and limitations of a creel tension control system using controlled disc brakes.

\section{MATERIAL AND METHOD}

A research stand was developed by using a single unit creel and a bobbin winding unit as shown in Figure 1.
A tensiometer of $200 \mathrm{cN}$ measuring interval was included in the research stand to measure yarn tension. Disc type of yarn brake was employed in the system and spring pressure was adjusted by a stepper motor in the disc brake to regulate yarn tension. Second stepper motor was included in the system to drive continuously the bottom disc. Motion is transmitted to the pressure spring via a worm and worm wheel and a screw mechanism. Bobbin diameter was measured by a laser sensor. Two stepper motors, the tension and laser sensors were interfaced to a PC and a software was developed in $\mathrm{C}$ programming language to collect data from tension and laser diameter sensors and to implement feedback tension control algorithms.

Figure 2 shows in more detail the pressure application mechanism in disc brake. The upper end of the spring is driven downwards by thee nut of screw mechanism in which the thread pitch is $0.8 \mathrm{~mm}$. Force induced in the spring due to compression affects as normal force to the upper plate of the disc brake. The motion is transmitted from the stepper motor to the screwed shaft via 1:16 gear ratio. Step motor used was able to run at maximum 300 rpm. Hence a spring pressure application speed of 0.25 $\mathrm{mm} / \mathrm{s}$ was possible.

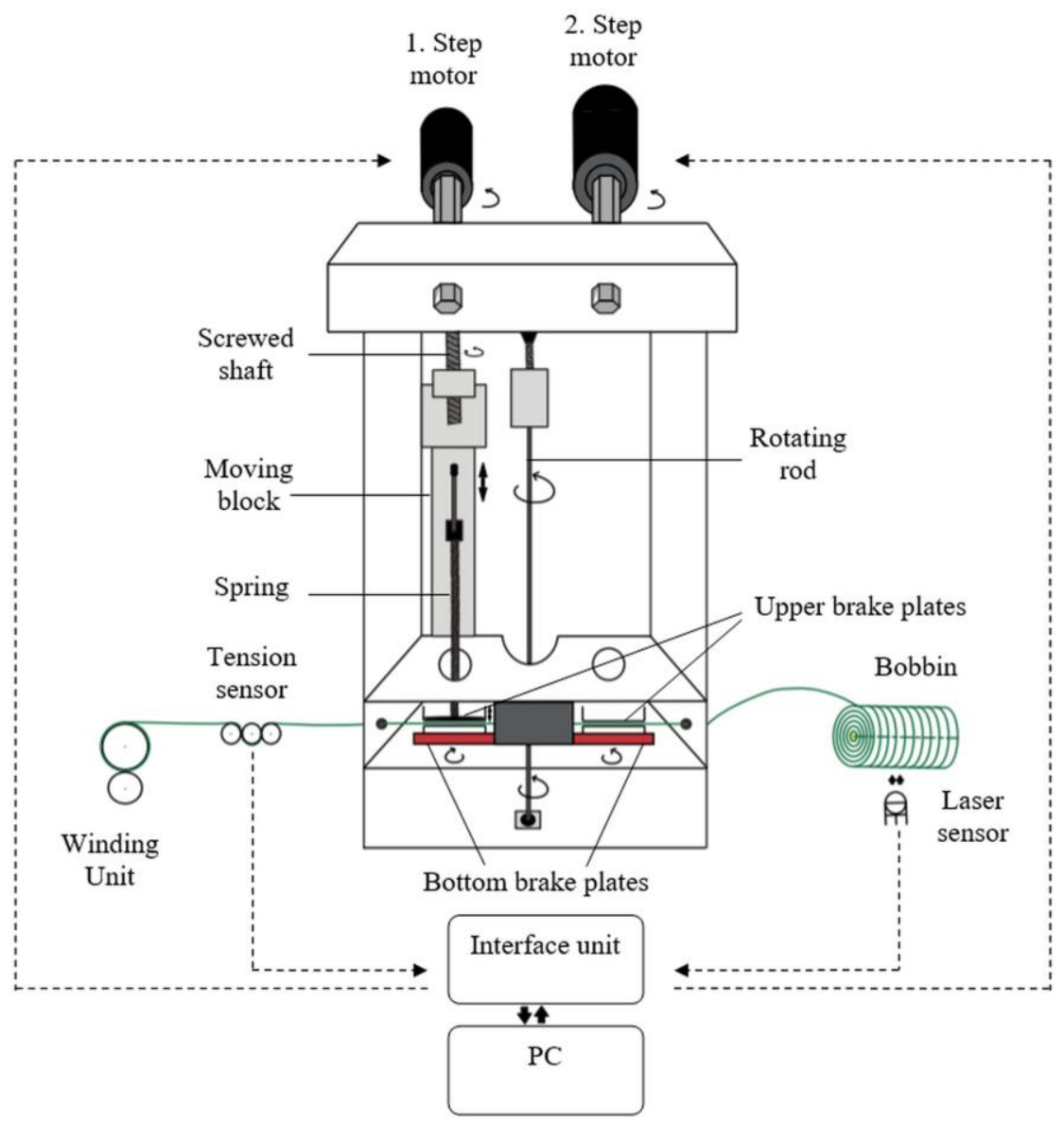

Figure 1. Experimental research stand 


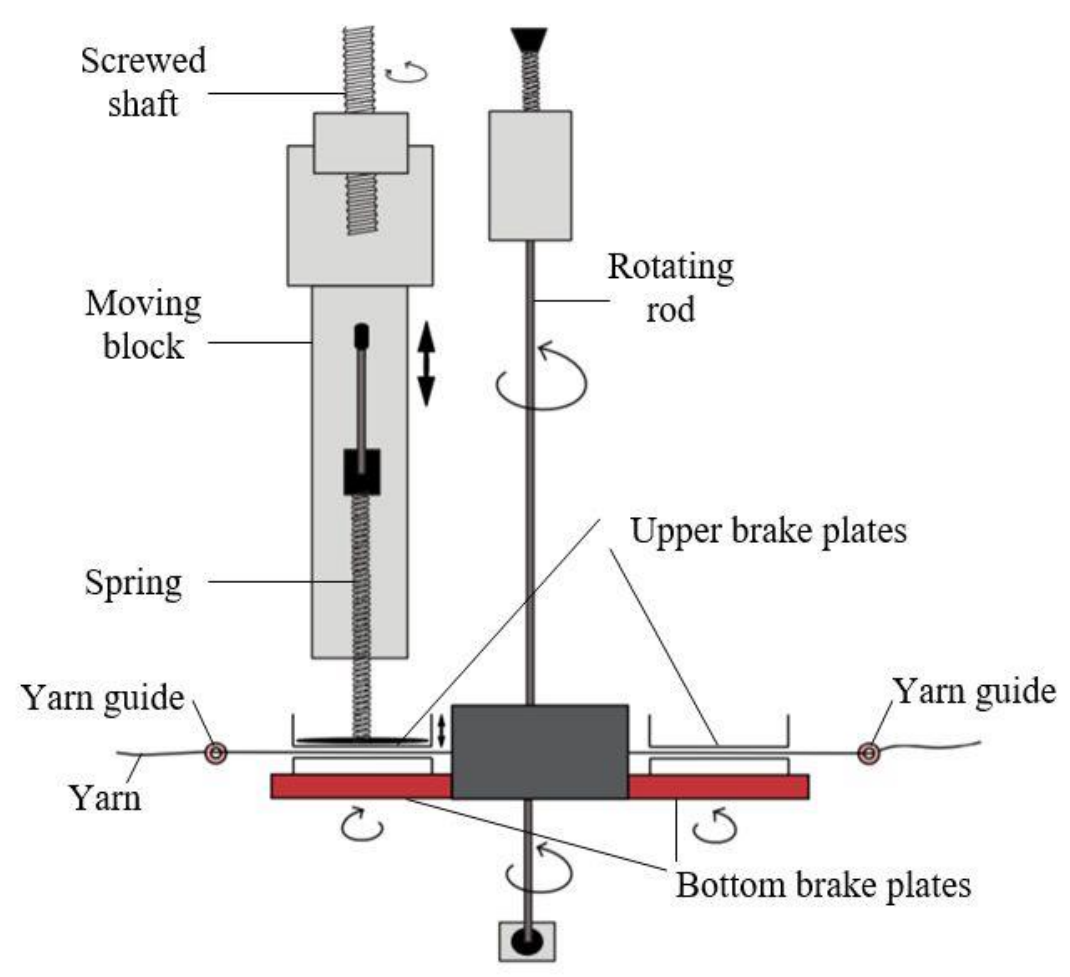

Figure 2. Pressure application mechanism in disc brake unit

To be able to develop and implement the feedback tension control system, yarn tension during unwinding needs to be analyzed. Figure 3 shows an example of typical yarn tension change during unwinding. As seen from the tension curve, yarn tension during unwinding fluctuates around its average value very significantly. There will always be tension fluctuations as in Figure 3 as the point of unwinding on bobbin surface shifts forward and backward during unwinding. Period of tension fluctuation is dependent on unwinding speed and bobbin and winding parameters. Therefore, it is difficult to determine a yarn length corresponding to one period of yarn tension chance. On the other hand, one period of tension change corresponding to around 1.25 meters of yarn in one type of bobbin takes 125 $\mathrm{msec}$ for $600 \mathrm{~m} / \mathrm{min}$ unwinding speeds. This period will be even shorter at speeds above $600 \mathrm{~m} / \mathrm{min}$. In warping machines, warp yarn is drawn from up to 800-1000 bobbins in sections. In this case, there will be a shift in tension curves due to different unwinding points on the different bobbins and total tension will be measured as the sum of all yarn tensions in a warp section. Therefore, total yarn tension change is expected to be different from single yarn tension change in both magnitude and shape and is not easy to predict. For this purpose, it will be a better strategy to apply a digital filter to the measured tension signal and obtain a stable tension value representing the average of tension change for automatic tension control purpose.

Experiments were carried out with 3 different cotton yarn counts. Yarn, winding and unwinding parameters used in the experimental work are presented in Table 1. Feedback tension control was applied at 330, 660 and $880 \mathrm{~m} / \mathrm{min}$ unwinding speeds with $\mathrm{Ne} 40 / 1,20 / 1$ and 10/1 cotton yarns from $200 \mathrm{~mm}$ full bobbin to $60 \mathrm{~mm}$ empty bobbin. Initially, all the bobbins were unwound at the specified speeds in Table 1 to determine warp tension variation from full to empty bobbin when braking was kept constant. Then, feedback tension control algorithms were implemented to keep yarn tension constant within the defined limits from full to empty bobbin. In implementing feedback tension control algorithms, initial average yarn tension was calculated over 25 tension values measured at $1.5 \mathrm{msec}$ intervals and then 10-values moving average tension was determined and compared with the desired yarn tension. A $\pm 0.5 \mathrm{cN}$ deviation in tension was allowed. When the moving average yarn tension reached above the desired one more than $0.5 \mathrm{cN}$, the stepper motor was driven in such a way as to decrease spring compression and release braking. In the case of the moving average tension decreasing more than $0.5 \mathrm{cN}$ compared to the desired yarn tension, the stepper motor was driven in opposite direction to increase the spring compression and therefore braking effect. Stepper motor of braking unit was not driven when the moving average tension stayed within $\pm 0.5 \mathrm{cN}$ of the desired tension. Stepper motor speed was not changed depending on the amount of deviation in tension; it was always driven at 2000 pulses/sec speed (around $300 \mathrm{rpm}$ ). Moving average approach was adopted in applying digital filtering to yarn tension data. For this purpose, yarn tension was measured at $1.5 \mathrm{msec}$ intervals and 25 of them were initially averaged. The yarn length over which the initial average yarn tension was calculated corresponds to 0.5 meter of yarn at $800 \mathrm{~m} / \mathrm{min}$ unwinding speed. Moving average tension was then calculated by using last initially averaged 10 and 50 tension values. When a last initially averaged tension is added as the first tension value, $10^{\text {th }}$ or 
$50^{\text {th }}$ tension value is removed and all the other initially averaged tension values are shifted one position further and new moving average tension is calculated. Figure 4 shows initially averaged yarn tension (grey) and 10- and 50-values moving averaged tension (black) curves from full to empty bobbin for Ne10/1 cotton yarn unwound at $880 \mathrm{~m} / \mathrm{min}$ speed. Comparison of Figure 4.a and 4.b shows clearly a better filtered tension curve with 50-values moving average. In this research, 10 -values moving average was used in forming feedback tension control system as it can be more responsive to even relatively shorter period average tension changes.

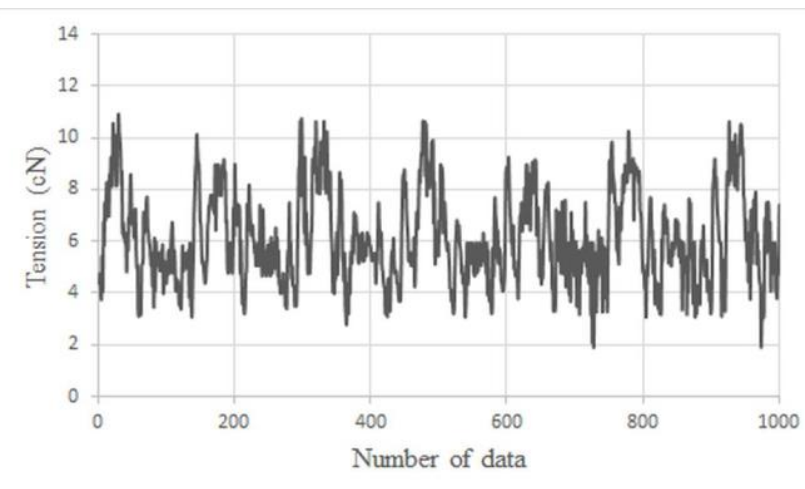

Figure 3. Yarn tension change during unwinding [10]

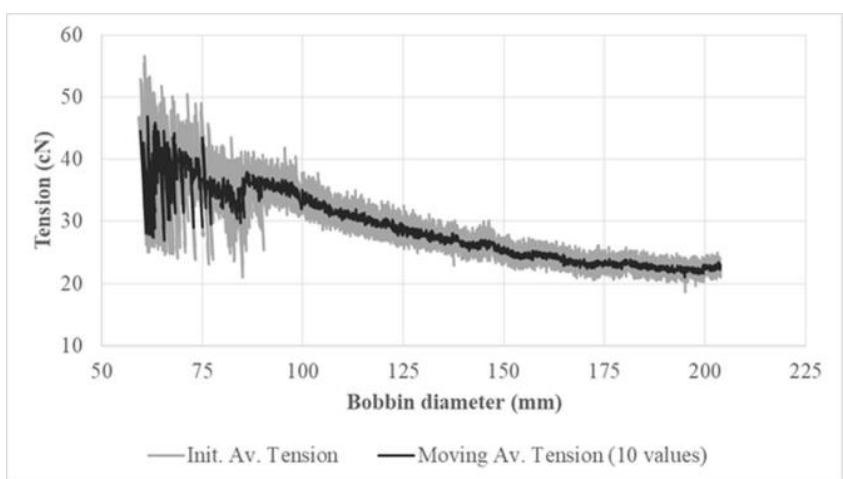

a. 10-values moving average curve

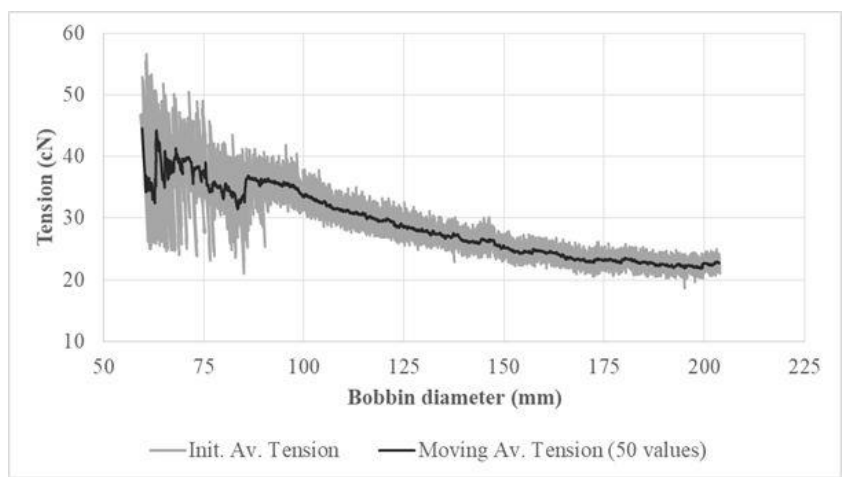

b. 50-values moving average curve

Figure 4. Initial averaged and moving averaged tension curves for $\mathrm{Ne} 10 / 1$ cotton yarn unwound at $880 \mathrm{~m} / \mathrm{min}$ speed

Table 1. Yarn, winding and unwinding parameters used in the experimental work

\begin{tabular}{ccccc}
\hline $\begin{array}{c}\text { Yarn type, } \\
\text { Winding type, } \\
\text { Bobbin shape }\end{array}$ & Yarn count & $\begin{array}{c}\text { Unwinding speed } \\
(\mathbf{m} / \mathbf{m i n})\end{array}$ & $\begin{array}{c}\text { Full bobbin } \\
\text { diameter } \\
(\mathbf{m m})\end{array}$ & $\begin{array}{c}\text { Empty bobbin } \\
\text { diameter } \\
(\mathbf{m m})\end{array}$ \\
\hline \multirow{2}{*}{ Cotton, } & Ne 10/1 & 330 & 200 & 60 \\
\cline { 2 - 5 } & & 660 & 200 & 60 \\
Random winding, & 880 & 200 & 60 \\
\hline Cylindrical & & 330 & 200 & 60 \\
& & 660 & 200 & 60 \\
& & 880 & 200 & 60 \\
\hline & & 330 & 200 & 60 \\
& & 660 & 200 & 60 \\
\hline
\end{tabular}

The method of the experimental study can be summarized as in Table 2 . 
Table 2. Summary of the experimental work

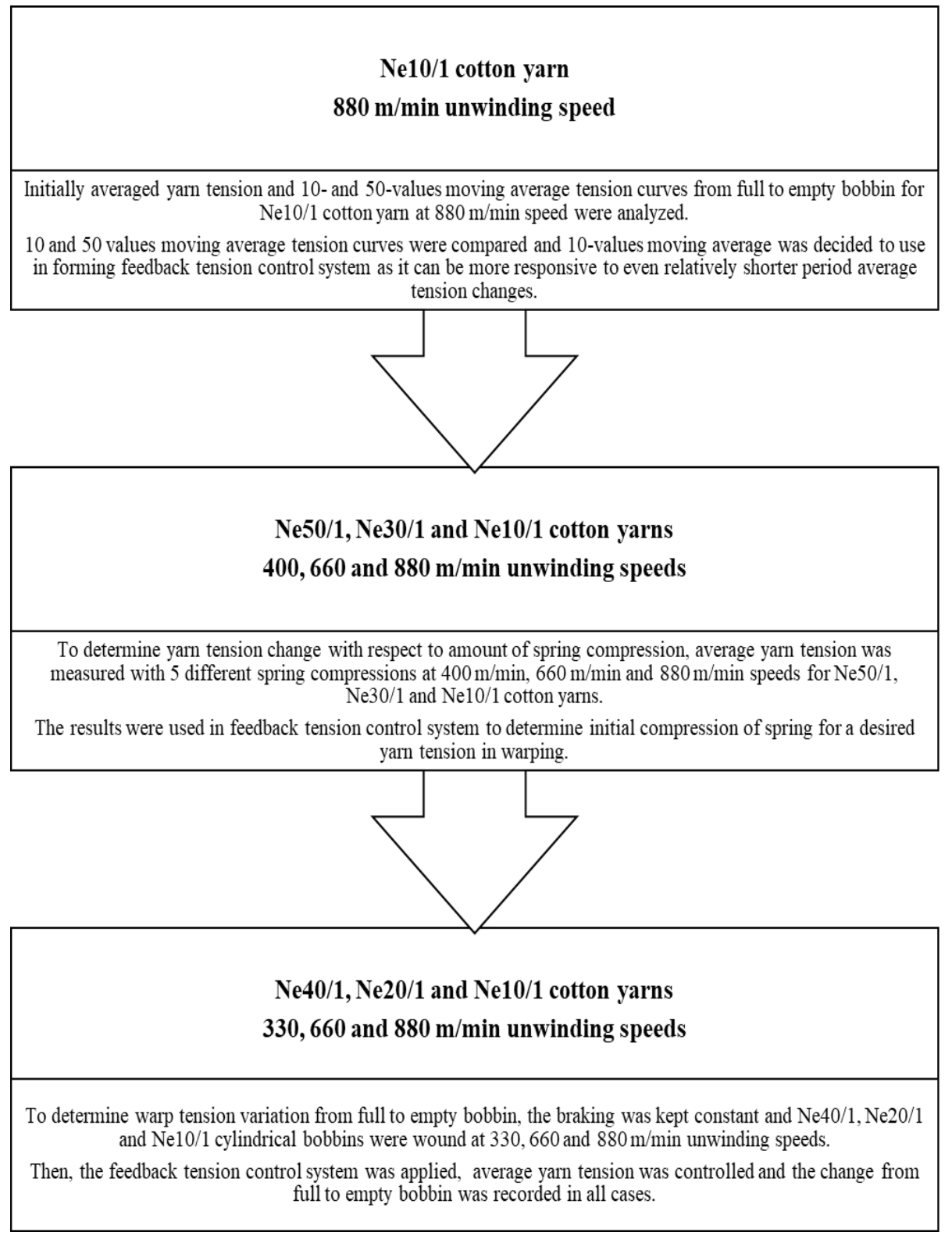

\section{RESULTS AND DISCUSSION}

Before implementing feedback tension control algorithms, experiments were conducted to determine yarn tension change with respect to amount of spring compression. Between 0 and $40 \mathrm{~mm}$, yarn tension was measured with 6000 successive readings of $1.5 \mathrm{msec}$ intervals and average tension was calculated in 5 different spring compressions at 400, 660 and $880 \mathrm{~m} / \mathrm{min}$ speeds for Ne50/1, 30/1 and 10/1 yarn counts. Figure 5.a, 5.b and 5.c show yarn tension change with respect to the amount of spring compression for $\mathrm{Ne} 10 / 1,30 / 1$ and 50/1, respectively. As seen from all figures, the average yarn tension increases almost linearly with the amount of compression. Slop of tension curves for different speeds is very close to each other which indicate very close amount of tension change with increasing spring compression irrespective of yarn unwinding speed. These results will be used in feedback tension control system to determine initial compression of spring for a desired yarn tension in warping. 


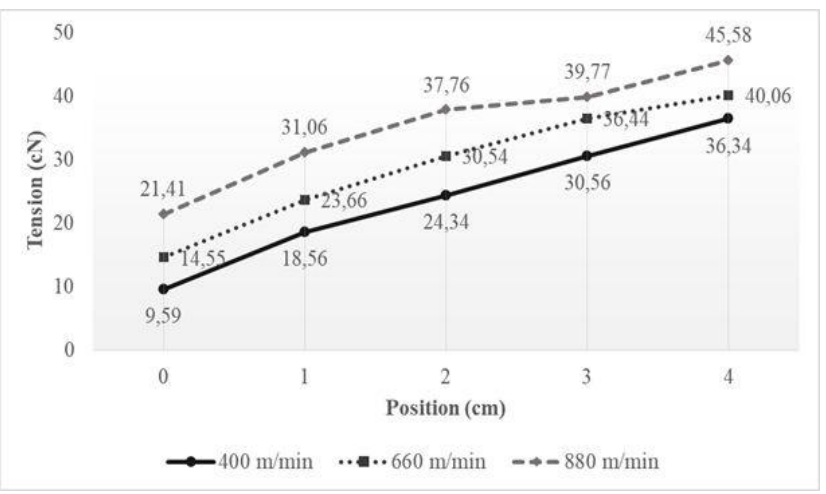

a. For Ne10/1 cotton yarn

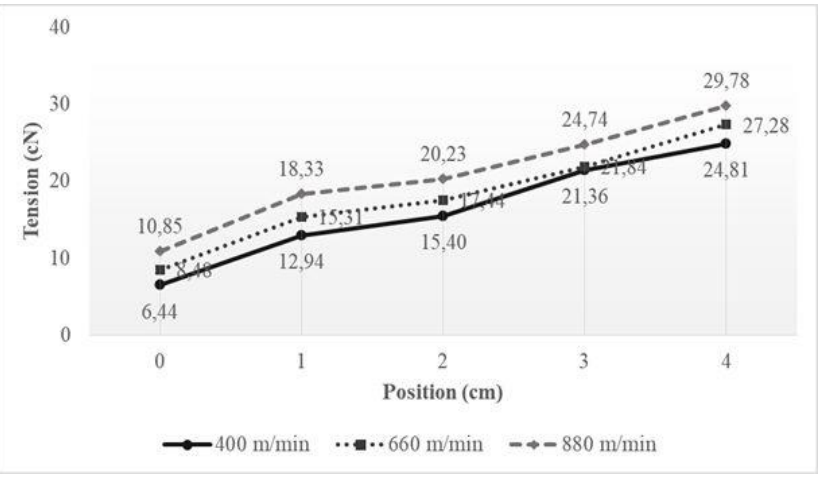

b. For Ne30/1 cotton yarn

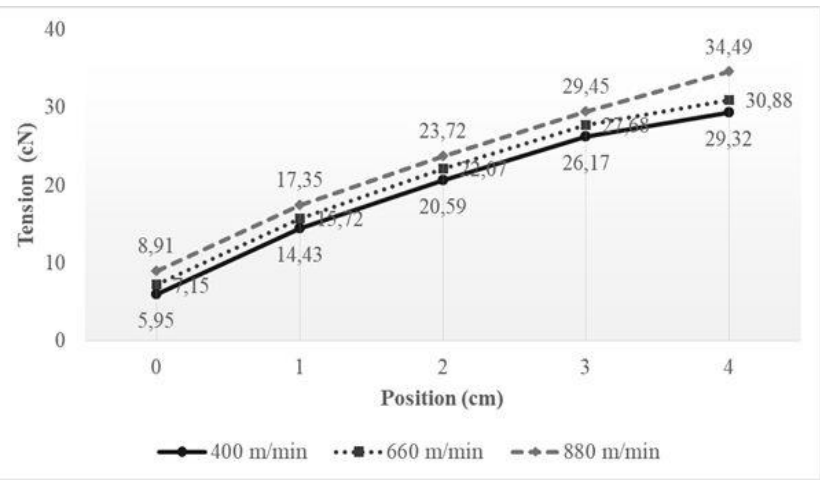

c. For Ne50/1 cotton yarn

Figure 5. Yarn tension change with respect to amount of spring compression

Figure 6.a, 6.b and 6.c show initial average (grey) and moving average (black) tension changes with respect to bobbin diameter for $\mathrm{Ne} 10 / 1$ cotton yarn at unwinding speeds of 330, 660 and $880 \mathrm{~m} / \mathrm{min}$, respectively. At 330 $\mathrm{m} / \mathrm{min}$ speed (Figure 6.a), there is around $8 \mathrm{cN}$ deviation in yarn tension from full to empty bobbin. Also, medium and relatively short-term tension variations are observed between 170 and $200 \mathrm{~mm}$ bobbin diameters. Relatively short term, medium term and long term (from full to empty bobbin) tension variations are significant from practical point of view and require a feedback tension control system. In the case of unwinding speeds of 660 and 880 $\mathrm{m} / \mathrm{min}$ speeds, yarn tension increases with few $\mathrm{cN}$ fluctuations (black curve) from full bobbin to 90 and 80 $\mathrm{mm}$ diameters for 660 and $880 \mathrm{~m} / \mathrm{min}$ speeds, respectively. From these diameters up to empty bobbin, there are large amount of tension fluctuations due to shift from to single to double balloon formation. Around $15 \mathrm{cN}$ tension increases from full to empty bobbin at $660 \mathrm{~m} / \mathrm{min}$ and $20 \mathrm{cN}$ at 880 $\mathrm{m} / \mathrm{min}$ speeds can be seen from Figure 6.b and 6.c. Also, short and medium term tension fluctuations of $10-15 \mathrm{cN}$ level occur towards the end of bobbins at both 660 and 880 $\mathrm{m} / \mathrm{min}$ speeds. Tension change and variations at this level can be very dangerous for both warping quality and efficiency and require control of tension.

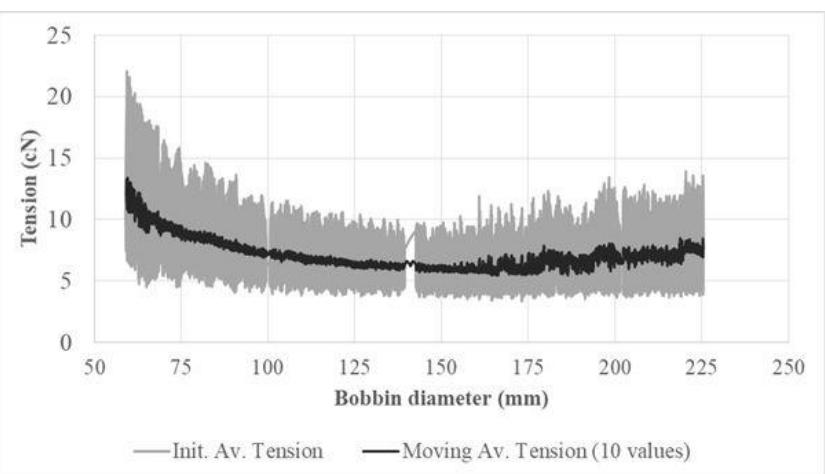

a. For $330 \mathrm{~m} / \mathrm{min}$ unwinding speed

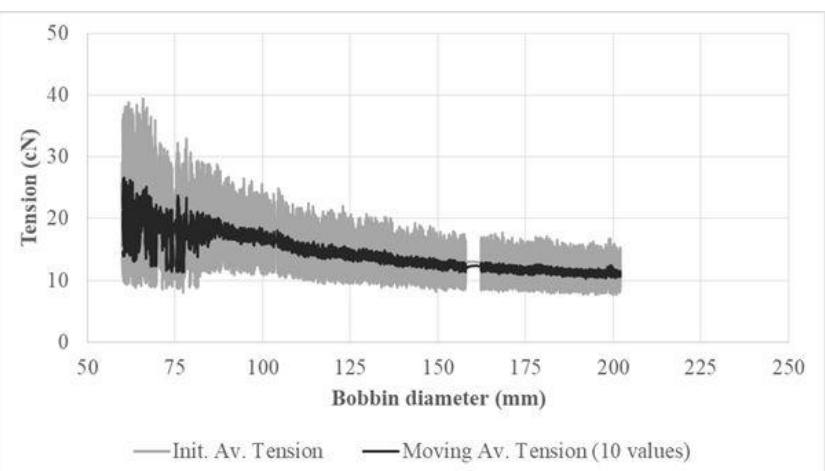

b. For $660 \mathrm{~m} / \mathrm{min}$ unwinding speed

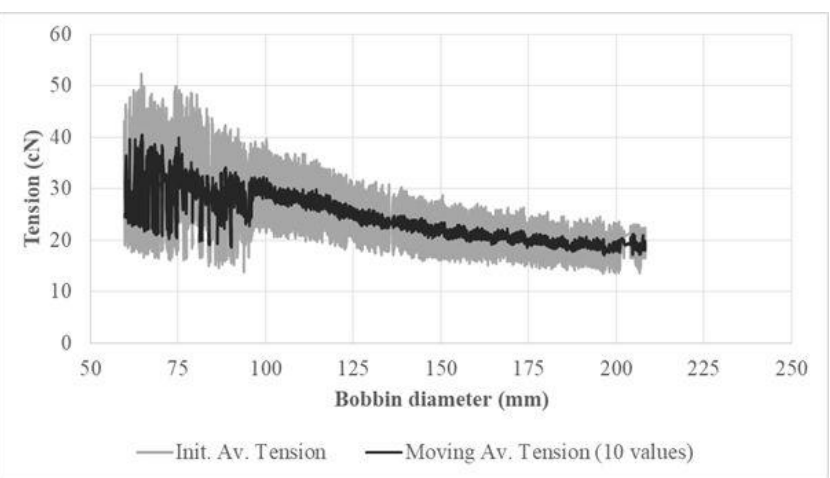

c. For $880 \mathrm{~m} / \mathrm{min}$ unwinding speed

Figure 6. Change of initial average (grey) and 10-values moving average (black) tensions with respect to bobbin diameter for $\mathrm{Ne} 10 / 1$ cotton yarn

Figure 7.a, 7.b and 7.c show yarn tension change during unwinding from full to empty bobbin at 330, 660 and 880 $\mathrm{m} /$ min speeds, respectively for $\mathrm{Ne} 20 / 1$ cotton yarn. At all unwinding speeds, yarn tension increases around $5 \mathrm{cN}$ at $330 \mathrm{~m} / \mathrm{min}, 9 \mathrm{cN}$ at $660 \mathrm{~m} / \mathrm{min}$ and $12 \mathrm{cN}$ at $880 \mathrm{~m} / \mathrm{min}$ 
speeds from full to empty bobbin. In some bobbin diameters, yarn tension shows short and medium term fluctuations amounting up to $5 \mathrm{cN}$. Amount of short, medium and long term (from full to empty bobbin) tension fluctuations is at unacceptable levels from practical point of view and harms the quality of warping. Short and medium term tension fluctuations are thought to be due to lower quality bobbin winding.

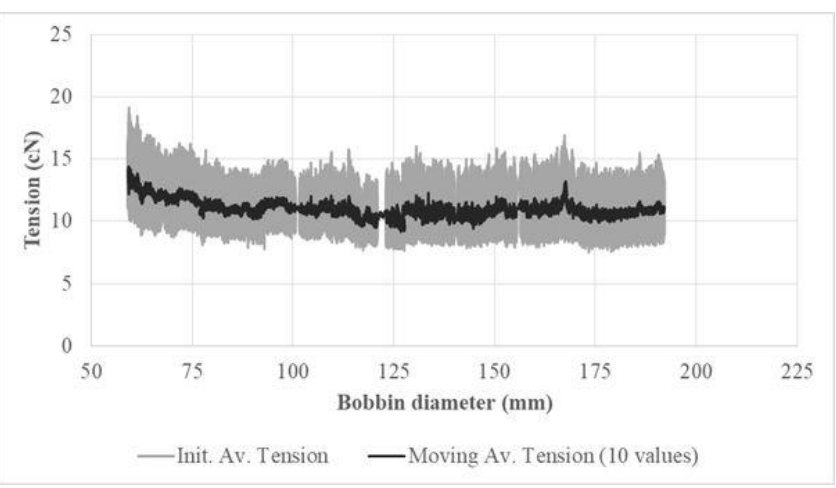

a. For $330 \mathrm{~m} / \mathrm{min}$ unwinding speed

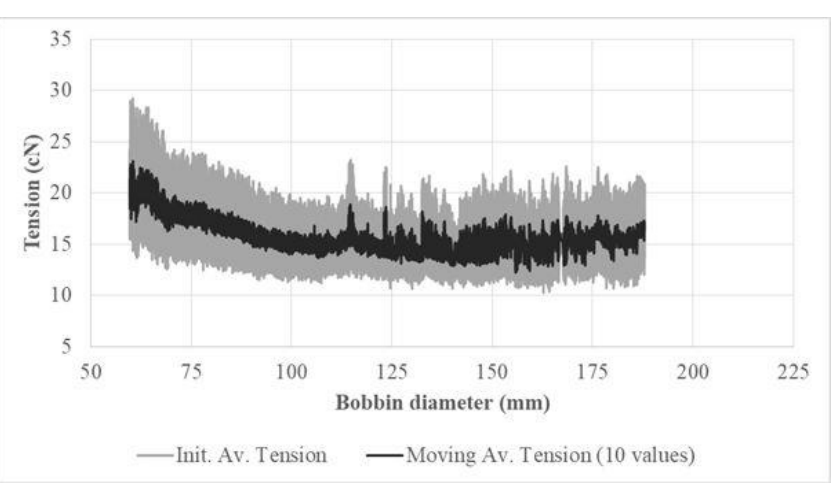

b. For $660 \mathrm{~m} / \mathrm{min}$ unwinding speed

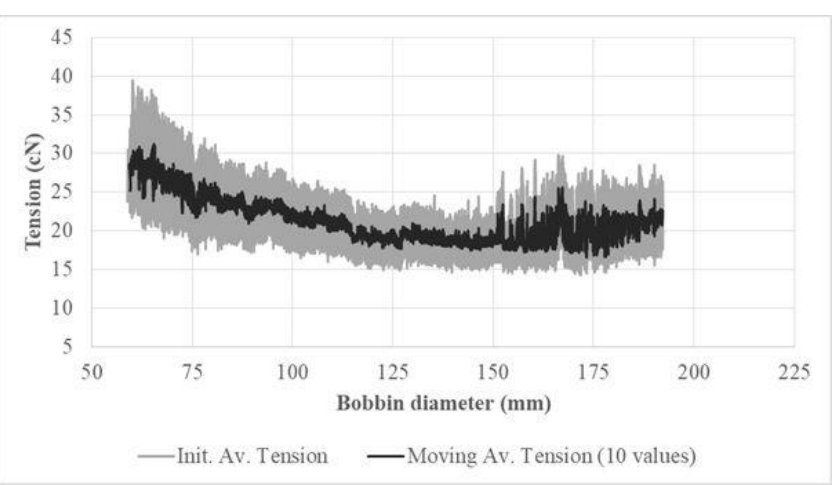

c. For $880 \mathrm{~m} / \mathrm{min}$ unwinding speed

Figure 7. Change of initial average (grey) and 10-values moving average (black) tensions with respect to bobbin diameter for Ne 20/1 cotton yarn

In the case of $\mathrm{Ne} 40 / 1$ cotton yarn, practically no significant tension change occurs at $330 \mathrm{~m} / \mathrm{min}$ unwinding speed (Figure 8.a). But, around $4 \mathrm{cN}$ tension change at $660 \mathrm{~m} / \mathrm{min}$ speed and $7 \mathrm{cN}$ tension change at $880 \mathrm{~m} / \mathrm{min}$ can be observed from Figure 8.b and Figure 8.c. Also, some tension fluctuations of short and medium term arise at 660 and $880 \mathrm{~m} / \mathrm{min}$ unwinding speeds.

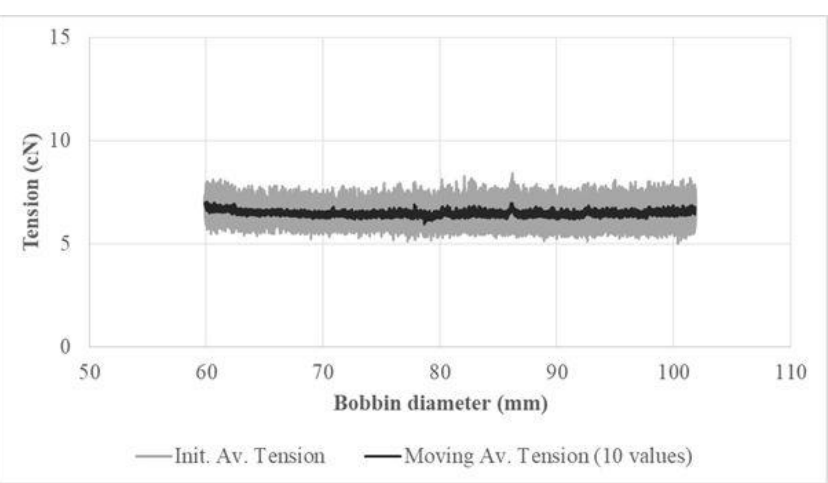

a. For $330 \mathrm{~m} / \mathrm{min}$ unwinding speed

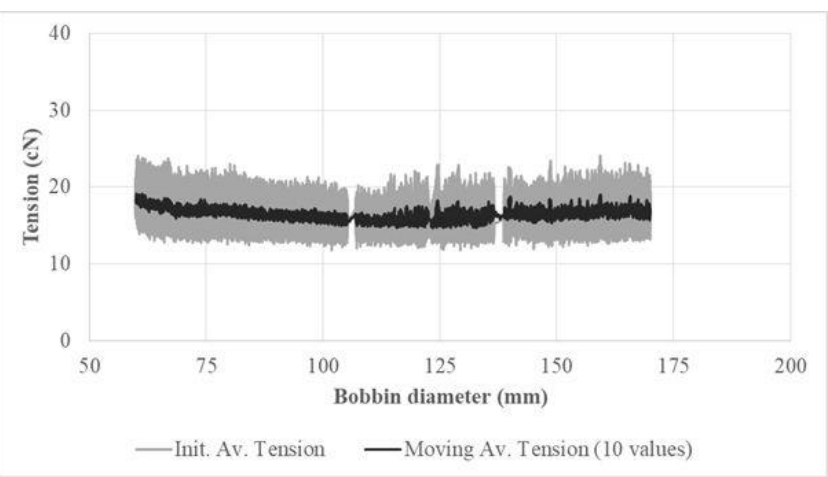

b. For $660 \mathrm{~m} / \mathrm{min}$ unwinding speed

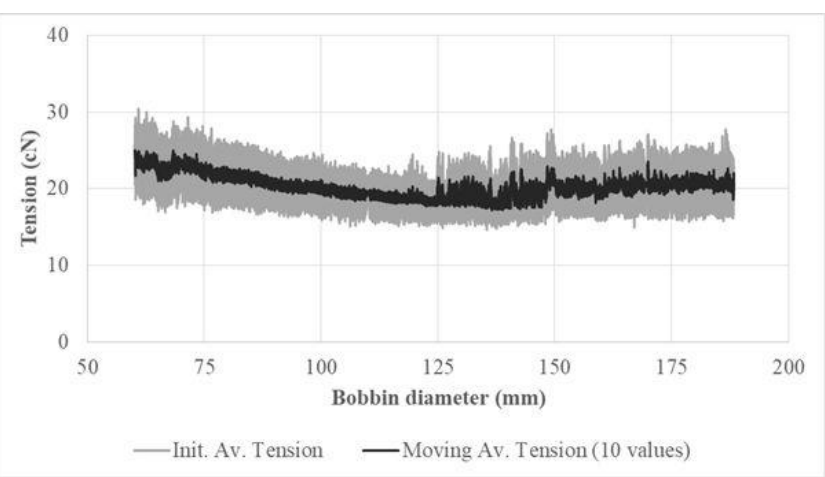

c. For $880 \mathrm{~m} / \mathrm{min}$ unwinding speed

Figure 8. Change of initial average (grey) and 10-values moving average (black) tensions with respect to bobbin diameter for $\mathrm{Ne} 40 / 1$ cotton yarn

The yarn tension change curves of Ne10/1, 20/1 and 40/1 yarns show that there are significant deviations from the initially adjusted yarn tension during unwinding at even lower speeds like $330 \mathrm{~m} / \mathrm{min}$. At speeds like 660 and 880 $\mathrm{m} / \mathrm{min}$ tension changes during unwinding are very significant and at harmfull level for both quality and efficiency of warping process. These results show the importance of using a feedback tension control system in warping creels.

Feedback tension control system was applied in a single unit creel by using $\mathrm{Ne} 40 / 1,20 / 1$ and 10/1 cylindrical bobbins at 330, 660 and $880 \mathrm{~m} / \mathrm{min}$ unwinding speeds and yarn tension change from full to empty bobbin was recorded in all cases. Feedback control system algorithms were applied as explained in "Material and Method" part. 
Figure 9.a, 9.b and 9.c show tension change from full to empty bobbin for Ne10/1 cotton yarn at 330, 660 and 880 $\mathrm{m} / \mathrm{min}$ speeds with feedback control algorithms applied. Yarn tension change turned to horizontal with a small fluctuation remaining within $\pm 1 \mathrm{cN}$ at 330 and $660 \mathrm{~m} / \mathrm{min}$ speeds (Figure 9.a and 9.b). At $880 \mathrm{~m} / \mathrm{min}$ speed, yarn tension showed the same change as in 330 and $660 \mathrm{~m} / \mathrm{min}$ speeds except the region corresponding last $90 \mathrm{~mm}$ bobbin diameter. In this region, yarn tension could be controlled with mostly $\pm 2 \mathrm{cN}$ fluctuation. This fluctuation is thought to be due to the higher tension fluctuations occurring without tension control presented in Figure 6.c.

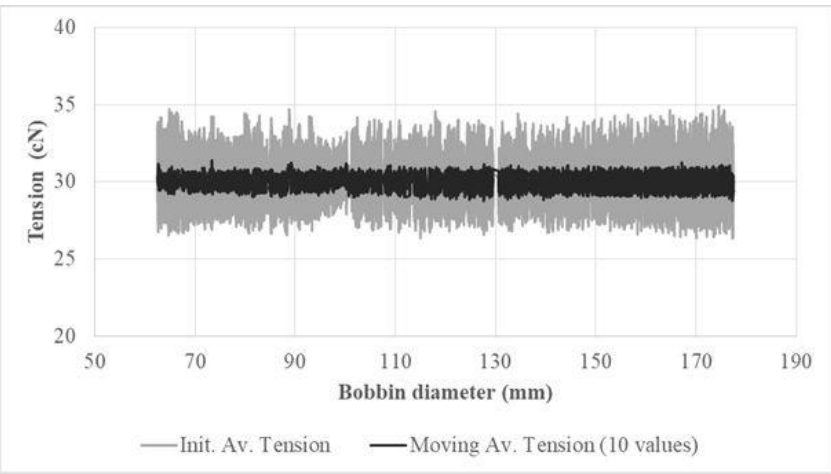

a. For $330 \mathrm{~m} / \mathrm{min}$ unwinding speed

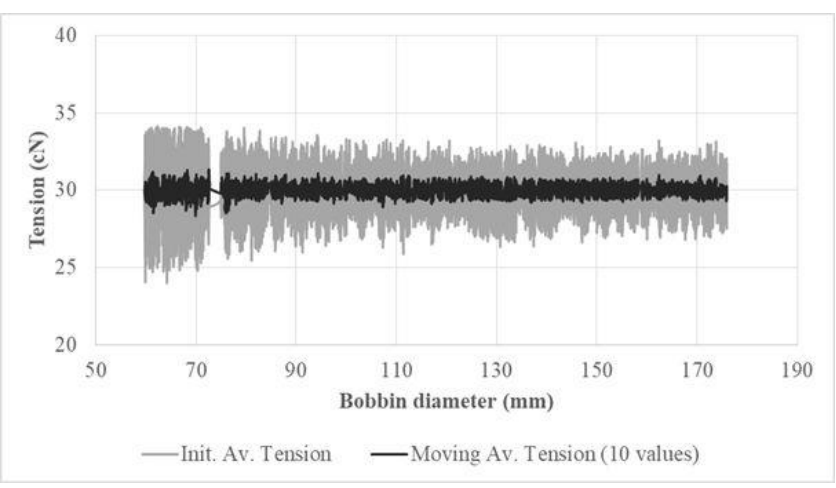

b. For $660 \mathrm{~m} / \mathrm{min}$ unwinding speed

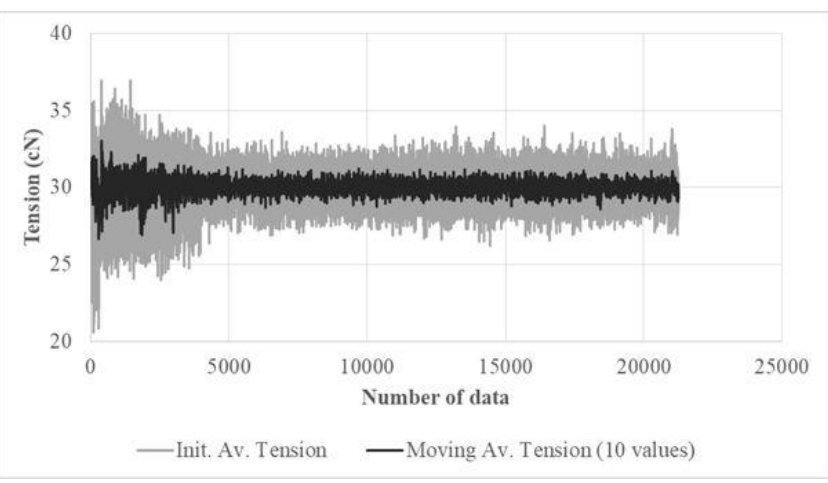

c. For $880 \mathrm{~m} / \mathrm{min}$ unwinding speed

Figure 9. Change of initial average (grey) and 10-values moving average (black) tensions with respect to bobbin diameter for $\mathrm{Ne}$

$10 / 1$ cotton yarn after applying feedback control algorithms

Figure 10 and Figure 11 show tension change from full to empty bobbin for $\mathrm{Ne} 20 / 1$ and 40/1 cotton yarn respectively at 330, 660 and $880 \mathrm{~m} / \mathrm{min}$ speeds with feedback control algorithms applied. As seen from all tension curves in
Figure 10 and Figure 11, yarn tension control was carried out at worst within $\pm 1 \mathrm{cN}$ with both yarn counts. In many cases, control system performance was even better than \pm 1 $\mathrm{cN}$.

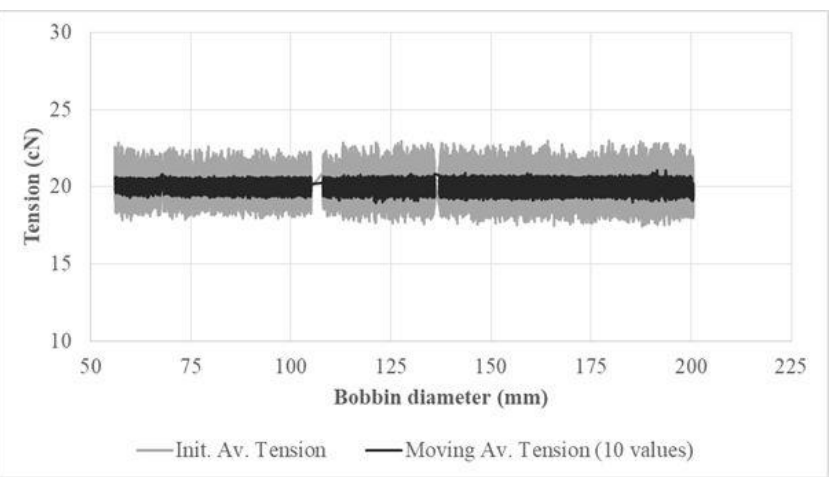

a. For $330 \mathrm{~m} / \mathrm{min}$ unwinding speed

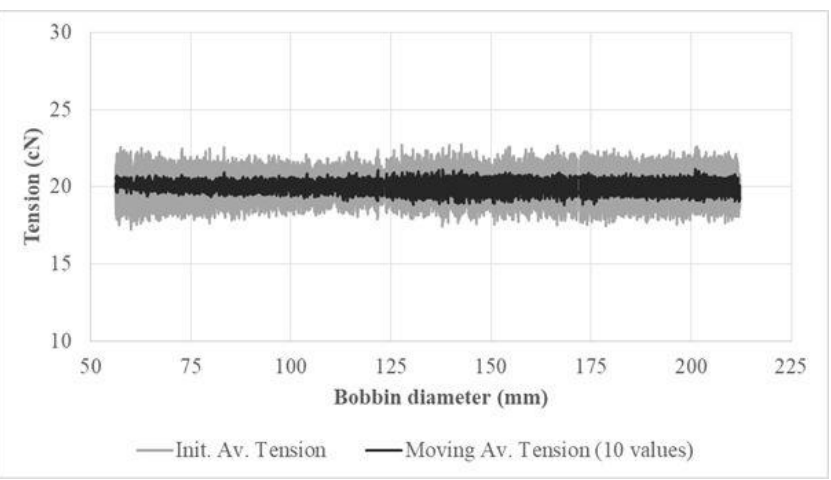

b. For $660 \mathrm{~m} / \mathrm{min}$ unwinding speed

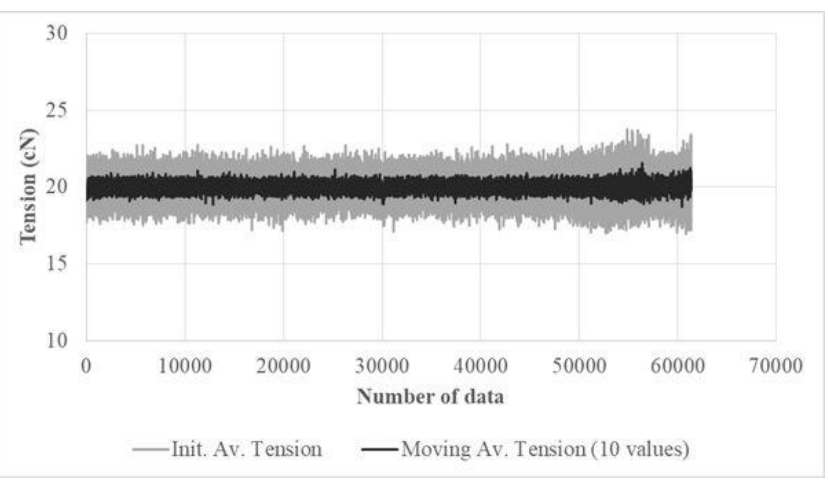

c. For $880 \mathrm{~m} / \mathrm{min}$ unwinding speed

Figure 10. Change of initial average (grey) and 10-values moving average (black) tensions with respect to bobbin diameter for $\mathrm{Ne} 20 / 1$ cotton yarn after applying feedback control algorithms

It should be noted here that yarn tension was measured at the exit of yarn brake and yarn contacted to only the yarn guide and yarn brake in this study. Therefore, some pressure was applied to yarn brake to obtain the desired tension even for thin yarn of $\mathrm{Ne} 40 / 1$. As there are certain number of contact points in creels and winding unit, yarn tension is increased due to frictional forces in contact points and there may not be any need for brake application in thin yarns to reach to the desired yarn tension for warping. At high speeds like over $500 \mathrm{~m} / \mathrm{min}$, practically significant 
tension variations can occur even with yarns requiring tension control application. This limits the application of feedback tension control system in warping machines with disc brakes.

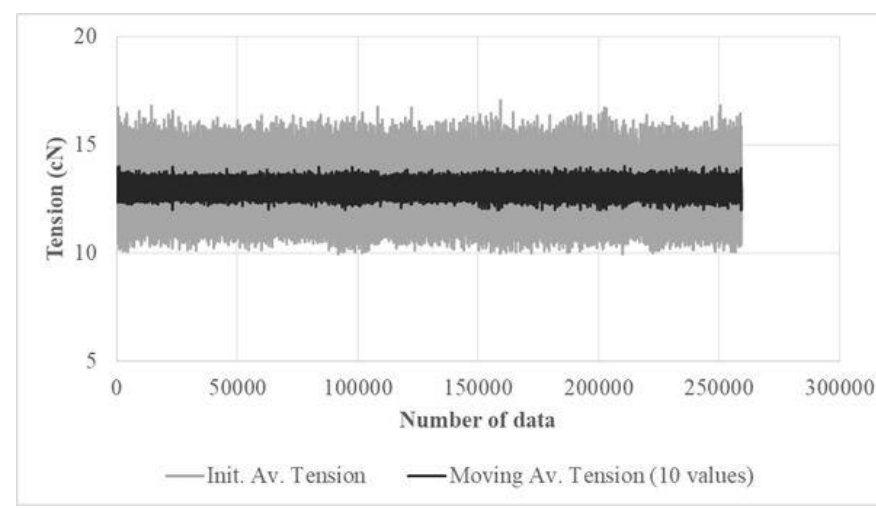

a. For $330 \mathrm{~m} / \mathrm{min}$ unwinding speed

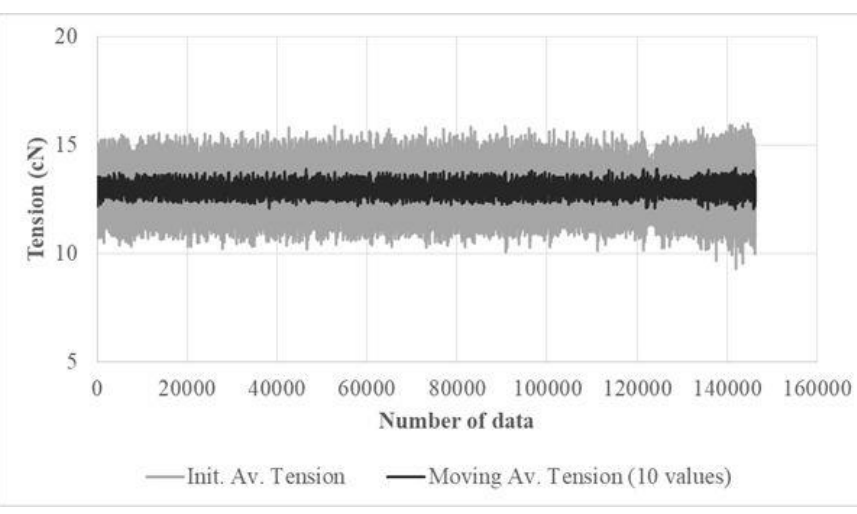

b. For $660 \mathrm{~m} / \mathrm{min}$ unwinding speed

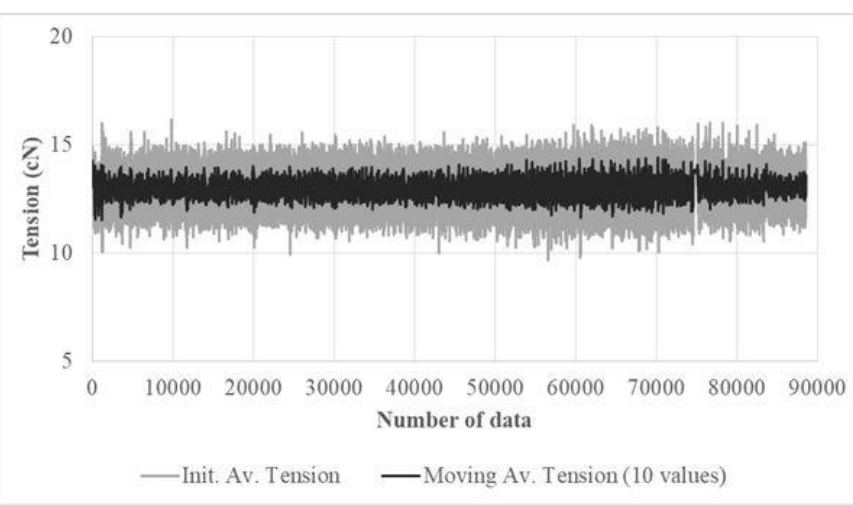

c. For $880 \mathrm{~m} / \mathrm{min}$ unwinding speed

Figure 11. Change of initial average (grey) and 10-values moving average (black) tensions with respect to bobbin diameter for Ne40/1 cotton yarn after applying feedback control algorithms

\section{CONCLUSION}

An experimental set up was developed to analyze yarn tension variations during unwinding from bobbins for warping process. Short, medium and long term yarn tension variations were observed at the significant level during unwinding of especially thicker yarns at higher speeds.
Depending on winding quality, relatively short or medium level tension variations were also recorded. At relatively high speeds for warping like 600 and $800 \mathrm{~m} / \mathrm{min}$, a significant yarn tension fluctuation occurred with thick yarns towards the empty bobbin (i.e., Ne 10/1) due to the shift from single to double balloon formation. Experimental results obtained with cotton yarns indicate the necessity for tension control in warping process at even moderate speeds like $300-400 \mathrm{~m} / \mathrm{min}$ for good quality of warp preparation.

A controlled disc brake was included in the single unit creel to form a feedback tension control system. Amount of brake was adjusted by increasing or decreasing the spring compression using a stepper motor. Stepper motor speed was not sufficient for quick response during stop period of warping machine. Therefore, it was focused mainly on controlling medium and long term tension variations. Feedback control algorithms were applied to Ne 40/1, 20/1 and 10/1 cotton yarns at 330, 660 and $880 \mathrm{~m} / \mathrm{min}$ speeds. It was shown that feedback tension control system successfully kept yarn tension at the adjusted value within $\pm 1 \mathrm{cN}$ from full to empty bobbin for most of the experiments. Control system performance showed some deviations like $\pm 2 \mathrm{cN}$ in experiments conducted with $\mathrm{Ne}$ $10 / 1$ cotton yarn at $880 \mathrm{~m} / \mathrm{min}$ unwinding speed in about last one third of full bobbin. Compared to tension fluctuations without feedback control, it can still be assessed as a good performance. In warp preparation with thicker yarns at high speeds, control system performance can be further improved by reducing unwinding speed towards empty bobbin.

In controlled brakes, control action is generated by decreasing braking with an increase in unwinding tension. There are many contact points on the yarn path between bobbin in the creel and winding head like cone drum. Friction in these contact points adds tension to the yarn and no brake application can be required for thin yarns at high speeds. In this case, tension increase during unwinding may not be compensated. This puts limitation to the use of controlled disc brakes for relatively thin yarns at high speeds. For yarns other than thin ones, sufficient amount of initial braking might be required from disc brake to compensate for yarn tension increase from full to empty bobbin.

Tension control system studied in this research does not include stop and start period of warping machines. As warping machines are suddenly stopped by applying a very strong brake yarn, tension of unwound yarns shows also a sudden decrease. Start period of a warping machine is much longer than stop period. Start and stop periods require application of the predetermined tension control algorithms to minimize tension variations for a short time interval and therefore necessitate a further study to develop the predetermined tension control algorithms. Research is in progress and will be the subject of another publication. 


\section{ACKNOWLEDGEMENT}

Main component of experimental set-up was developed in the project (Project Number: 215M372) supported by The Scientific and Technological Research Council of Turkey

\section{REFERENCES}

1. Eren R. 2009. Dokuma Hazırlı Teknolojisi. Bursa: MKM Yayınları.

2. Karl Mayer. 2019, March 20. Yarn tensioners/ yarn stop motions. Retrieved from https://www.karlmayer.com/en/products/warppreparation/creels/yarn-tensioners-yarn-stop-motions/.

3. Fernando E, Kuruppu RU. 2015. Tension Variation in sectional warping, Part I: Mathematical modeling of yarn tension in a creel International Journal of Engineering and Advanced Technolog 4(3), 158-164.

4. Isakov NP. 1961. Yarn tension in a balloon. Tech. of Textile Industry U.S.S.R. 2, 92-98.

5. Dorgham ME. 2013. Warping parameters influence on warp yarns properties: Part 1: Warping speed and warp yarn tension. Journal of Textile Science and Engineering 3(132), doi:10.4172/21658064.1000132.
(TÜBİTAK). We would like to express our sincere thanks to TÜBITAK for supporting this project. Also, we would like to thank to Devsan Makina Tekstil San. Tic. Ltd. Şti. for manufacturing step motor drive unit of yarn brake.

6. Beerli M, Guntli E. 1989. Apparatus for regulating the warp section tension during warping.US4819310A. Place:US Patent.

7. Zeller HP, Bollen M, Spari A, Hane S. 2003. Process for the operation of a bobbin creel and bobbin creel for a winding system. US6513748B2. Place:US Patent.

8. Kleiner, A., Jakob, A. 2010. Method and device for operating a creel designed for a winding system and corresponding creel. US7770271B2. Place:US Patent.

9. Baba Y. 1991. Tension controller for warping machine and warping method. US5027484A. Place:US Patent.

10. Çelik Ö. 2018. Bobin sağımında iplik gerginliğine etki eden faktörlerin deneysel araștırılması (Doctoral dissertation). Bursa Uludag University Graduate School of Natural and App. Science Textile Engineering Department, Bursa. 